\title{
A computational study of the effect of windscreen shape and flow resistivity on turbulent wind noise reduction
}

\author{
Ying Xu \\ Key Laboratory of Microgravity, Institute of Mechanics, Chinese Academy of Science, Beijing, 100190, China
}

\author{
Z. C. Zheng ${ }^{\text {a) }}$ \\ Department of Aerospace Engineering, University of Kansas, Lawrence, Kansas 66045 \\ D. K. Wilson \\ U.S. Army Cold Regions Research and Engineering Laboratory, Engineering Research and Development \\ Center, Hanover, New Hampshire 03755
}

(Received 10 June 2010; revised 13 January 2011; accepted 15 January 2011)

\begin{abstract}
In this paper, numerical simulations are used to study the turbulent wind noise reduction effect of microphone windscreens with varying shapes and flow resistivities. Typical windscreen shapes consisting of circular, elliptical, and rectangular cylinders are investigated. A turbulent environment is generated by placing a solid circular cylinder upstream of the microphone. An immersed-boundary method with a fifth-order weighted essentially non-oscillatory scheme is implemented to enhance the simulation accuracy for high-Reynolds number flow around the solid cylinder as well as at the interface between the open air and the porous material comprising the windscreen. The Navier-Stokes equations for incompressible flow are solved in the open air. For the flow inside the porous material, a modified form of the Zwikker-Kosten equation is solved. The results show that, on average, the circular and horizontal ellipse windscreens have similar overall wind noise reduction performance, while the horizontal ellipse windscreen with medium flow resistivity provides the most effective wind noise reduction among all the considered cases. The vertical ellipse windscreen with high flow resistivity, in particular, increases the wind noise because of increased self-generation of turbulence.
\end{abstract}

(C) 2011 Acoustical Society of America. [DOI: 10.1121/1.3552886]

PACS number(s): 43.28.Gq, 43.28.Ra [VEO]

Pages: $1740-1747$

\section{INTRODUCTION}

When microphones are used for outdoor acoustic measurements, wind noise interferes with the signals, particularly in the low-frequency range. Wind noise is due to turbulent pressure fluctuations occurring on a microphone. Windscreens are thus used on microphones to attenuate wind noise. Effective wind noise reduction (WNR) depends on correct designs of windscreen materials and shapes. As production and reduction of wind noise inside a windscreen is a complicated aerodynamic problem, optimized windscreen designs are difficult to predict by theoretical methods.

In this study, the shape effect of windscreens is investigated by comparing windscreen shapes consisting of circular, elliptical, and rectangular cylinders. Effects of turbulence and the porous windscreen material properties, specifically its flow resistivity, are also investigated. Time-domain computational techniques are developed to study the detailed flow mechanisms around the windscreen as well as the flow inside the windscreen.

In an early study of microphone wind noise, Strasberg $^{1}$ employed a dimensional analysis and experimental data in wind tunnels to determine if the pressure within a spherical or cylindrical wind screen with diameter $D$ in a flow with velocity $U$ depended on the non-dimensional characteristic pa-

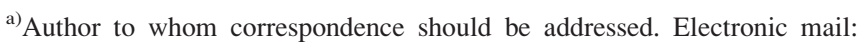
zzheng@ku.edu
}

rameters such as the Reynolds number $R e=D U / v$, where $v$ is the kinematic viscosity of air, the Strouhal number $S_{r}=f D / U$, where $f$ is the frequency, and the Mach number $M=U / c$, where $c$ is the speed of sound in the air. He concluded that, for a quiescent incoming flow, only Strouhal number was a strong factor.

Morgan and Raspet ${ }^{2}$ measured the wind noise and the instantaneous wind speed for bare and screened microphones in an outdoor environment. They stated that, for high turbulence conditions, the dominant source of pressure fluctuations at the microphone outdoors is the intrinsic turbulence in the incoming flow.

An early model of windscreen noise reduction at very low wind turbulence frequency was that of a rigid smooth sphere with impermeable surface transmitting the pressure fluctuations to quiescent media inside the sphere. ${ }^{3,4}$ That model was restricted to flow turbulence with scales much larger than the sphere diameter. The mean flow across the sphere could thus be considered steady. The pressure fluctuations induced on the spherical surface, the distribution of which coincided mathematically with that of the corresponding steady surface pressure coefficient, were then used to obtain the pressure levels that could be sensed by a microphone placed at the center of the sphere. This was because pressure fluctuations inside the windscreen were assumed to satisfy the Laplace equation. The justification of the use of a steady-state flow model was based on experimental data by Morgan $^{5}$ and Morgan and Raspet. ${ }^{2}$ They showed that when 
the screen number $D / \lambda$ (the ratio between the sphere diameter and the wavelength) was below 0.3 (the corresponding frequency was about below $10 \mathrm{~Hz}$ ), the noise reduction sensed at the center was almost constant. Beyond that ratio, a rapid rise with the decrease of wavelength appeared, because the scale of the turbulence became smaller and the steady-state assumption was no longer valid.

The strength of wind noise was scaled in Morgan and Raspet's work by $\rho\left|U u^{\prime}\right|$, where $\rho$ is the density of the air, $U$ is the speed of the incoming wind, and $u^{\prime}$ is the velocity fluctuation in the direction of the incoming wind. This scaling was deduced from the Bernoulli relation for a stagnation pressure of

$$
P_{s}=\frac{1}{2} \rho u^{2}
$$

where $u=U+u^{\prime}$. In addition, the condition $|U| \gg\left|u^{\prime}\right|$ must be satisfied so that the contribution $(1 / 2) \rho\left|u^{\prime}\right|^{2}$ could be neglected in comparison to $\rho\left|U u^{\prime}\right|$.

Since wind noise can be represented nominally by $\rho\left|U u^{\prime}\right|$, Raspet et al. ${ }^{6}$ and van den Berg ${ }^{7}$ provided more detailed theoretical understanding and mechanisms of atmospheric turbulence effects on wind noise by examining the turbulent velocity correlation and turbulent kinetic energy spectra. However, Raspet et al. ${ }^{6}$ pointed out that there is another wind noise source due to interaction between the windscreen and the flow. The generated pressure fluctuations around the windscreen produce self-noise. Since this self-noise is caused by interactions between the flow and the windscreen, it is thus expected to closely relate to the windscreen shape.

Time-domain numerical simulation methods ${ }^{8,9}$ can be combined with computational fluid dynamics to provide powerful new tools to tackle acoustical problems. Recently, a finite-difference, time-domain (FDTD) method was developed to simulate turbulence-induced pressure fluctuations around a porous microphone windscreen. ${ }^{10}$ In this approach, the unsteady, incompressible fluid flow equations were solved for the air flow, whereas an incompressible form of the Zwikker-Kosten $(\mathrm{ZK})^{11}$ equation was solved within the porous medium under low-Reynolds number flow condition with a third-order upwind scheme implemented around the interface zone between the air and the porous medium. The simulation results showed that, for low-frequency turbulence, windscreens with low flow resistivity were more effective in noise reduction, while for high-frequency turbulence, windscreens with high flow resistivity were more effective. In this paper, a high-order (fifth-order), weighted essentially nonoscillatory (WENO) scheme coupled simulation is used for solving these same equations to study the windscreen shape effect, along with the flow resistivity effect, on WNR under a high-Reynolds number flow condition.

The discontinuity at the interface between the fluid and the porous medium has been found detrimental to computational accuracy. The accuracy at the interface is particularly important for flow with a high-Reynolds number. One of the most effective ways to overcome the discontinuity is to apply high-order schemes. ${ }^{10}$ Hence, in this study, a WENO scheme ${ }^{12,13}$ is used in the regions near the interface between the fluid and porous media and is combined with a modified immersed-boundary (IB) method ${ }^{14,15}$ to solve the governing equations. The use of a WENO scheme enables simulation of high-Reynolds number flow through different media; this is important, as Reynolds numbers for atmospheric turbulence are ordinarily quite large $\left[\mathrm{O}\left(10^{5}\right)\right.$ or greater]. Broadband pressure fluctuations are generated by placing a solid circular cylinder upstream of the microphone, which produces wake turbulence corresponding to a cylinder-diameter based Reynolds number of 5000. Although more realistic atmospheric turbulence could be incorporated in future studies using other methods such as the quasi-wavelet fields, ${ }^{16,17}$ such methods may require further improvements if they are to be adapted for numerical simulation. Nevertheless, this study provides a significant step in solving the numerically and physically complex problem of turbulent WNR.

\section{FORMULATION AND SOLUTION SCHEMES}

The assumed, two-dimensional model problem is shown in Fig. 1. A uniform flow passes around an upstream solid cylinder that generates a stream of unsteady and/or turbulent flow approaching an unscreened or a screened microphone located downstream. The windscreen, when present, is made of a porous material. Unsteady pressure fluctuations are generated by flow fluctuations and vortical structures around the surface of the windscreen and in the wake region. Such pressure fluctuations sensed by the microphone, which is assumed to be the pressure at the center of the cylinder representing the microphone, result from near-field, incompressible disturbances. The flow fluctuations, both internal and external to the windscreen, have been investigated with a coupled flow simulation between the outside and inside of the windscreen utilizing an IB method ${ }^{10}$ on a Cartesian grid, as the IB methods for fluid-structure interaction problems typically discretize the equations of motion for fluid on a Cartesian grid. ${ }^{14,15}$

The model equations are the Navier-Stokes (NS) equations for incompressible flow outside the porous medium, with a modified $\mathrm{ZK}$ equation ${ }^{11}$ for flow inside the porous

(a)
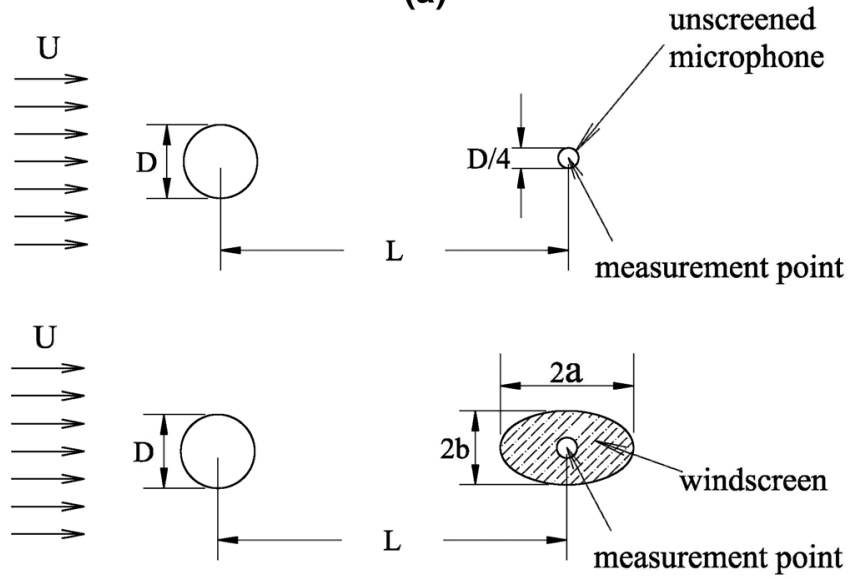

(b)

FIG. 1. Illustration of the model problem and the computational domain: (a) an unscreened microphone and (b) a screened microphone. 
medium. Although sound waves measured at a microphone are compressible disturbances, the wind noise interfering with the sound waves consists, in general, of incompressible turbulence. The pressure fluctuations of interest are near the surface of an object or inside a porous medium. These fluctuations are associated with near-field, as with the surface pressure fluctuations produced by a turbulent boundary layer over the surface (as discussed by Kraichnan ${ }^{18}$ ), for which the incompressible flow assumption is well justified. The enhancement and reduction of the near-field pressure fluctuations are then interpreted as the effect of windscreen on wind noise in this study.

The governing equations are non-dimensionalized with the incoming wind speed, $U$, the diameter of the upstream cylinder, $D$, and the air density, $\rho$, to become

$$
\frac{\partial u_{i}}{\partial t}+\frac{\partial}{\partial x_{j}}\left(u_{i} u_{j}\right)=-\frac{\partial p}{\partial x_{i}}+\frac{1}{\operatorname{Re}} \frac{\partial^{2} u_{i}}{\partial x_{j} \partial x_{j}}+f_{i},
$$

and

$$
\frac{\partial u_{j}}{\partial x_{j}}=0
$$

where all the variables are dimensionless, and the indices $i, j=1,2$, follow the Einstein summation convection with summations assumed over repeated indices. The body force, $f_{i}$, is a fictitious force inducing the flow to accommodate that inside the porous or solid cylinder. ${ }^{14}$

We begin by discretizing the momentum equation [Eq. (2)] as

$$
\frac{u_{i}^{n+1}-u_{i}^{n}}{\Delta t}=\mathrm{RHS}_{i}+f_{i},
$$

where

$$
\mathrm{RHS}_{i}=-\frac{\partial}{\partial x_{j}}\left(u_{i} u_{j}\right)-\frac{\partial p}{\partial x_{i}}+\frac{1}{\operatorname{Re}} \frac{\partial^{2} u_{i}}{\partial x_{j} \partial x_{j}}
$$

and the $f_{i}$ is given by

$$
f_{i}=\left\{\begin{array}{cl}
0 & \text { outside windscreen } \\
\sigma u_{i} & \text { inside the windscreen }, \\
-\mathrm{RHS}_{1}+\left(v_{b i}^{n+1}-u_{i}^{n}\right) / \Delta t & \text { inside solid body }
\end{array}\right.
$$

where $v_{b i}^{n+1}$ is the velocity of the solid body at the $n+1$ time step. This formulation assures that the condition $u_{b i}^{n+1}=v_{b i}^{n+1}$ will be satisfied inside the solid body.

In the case of flow inside a porous windscreen, the porosity and structure constant are assumed to equal 1 for simplicity. The governing equations for airflow inside the windscreen are expressed in Eqs. (2) and (3) as $f_{i}=\sigma u_{i}$, where $\sigma$ is the dimensionless flow resistivity of the porous medium, non-dimensionalized by $\rho U / D$. The resulting momentum equation for air flow inside the windscreen is the $\mathrm{ZK}$ equation, ${ }^{11}$ which is the low-frequency limit of more general forms of porous media equations. ${ }^{19}$ In addition, incompressibility is assumed for flow inside the porous me- dium. The convection and diffusion terms are neglected in the original $\mathrm{ZK}$ equation because the velocity is low in the porous medium. We nonetheless retain them here so that the same solver can be used for both the NS equation and ZK equation, as the effect of convection and diffusion automatically becomes small when the velocity is low. The nonlinear drag effect caused by the second-order velocity, i.e., the Forchheimer term, is neglected in this study.

The presence of the porous medium introduces a discontinuity in some of the flow variables or their derivatives around the flow/porous interface. Under these circumstances most conventional finite-difference schemes would generate spurious numerical oscillations. To avoid such oscillations, we apply a fifth-order WENO scheme.

The fifth-order WENO scheme can be introduced by considering the simplified one-dimensional, one-way factored wave equation in the form of

$$
\frac{\partial u}{\partial t}+\frac{\partial q(u)}{\partial x}=0
$$

The derivative of any flux, $q$, is discretized as

$$
\left.\frac{\partial q}{\partial x}\right|_{x-x_{i}}=q_{x, i}=\frac{\hat{q}_{i=1 / 2}-\hat{q}_{i-1 / 2}}{\Delta x}
$$

where $\hat{q}_{i+1 / 2}$ is an interpolated flux at the half-step location.

$$
\text { If } \partial q / \partial u \geq 0 \text {, }
$$

$$
\begin{aligned}
\hat{q}_{i+1 / 2}^{+}= & \omega_{1}\left(\frac{1}{3} q_{i-2}-\frac{7}{6} q_{i-1}+\frac{11}{6} q_{i}\right) \\
& +\omega_{2}\left(-\frac{1}{6} q_{i-1}+\frac{5}{6} q_{i}+\frac{1}{3} q_{i+1}\right) \\
& +\omega_{3}\left(\frac{1}{3} q_{i}+\frac{5}{6} q_{i+1}-\frac{1}{6} q_{i+2}\right)
\end{aligned}
$$

where

$$
\begin{aligned}
& d_{1}=\frac{1}{10}, \quad d_{2}=\frac{3}{5}, \quad d_{3}=\frac{3}{10}, \quad \varepsilon=10^{-6}, \\
& \beta_{1}=\frac{13}{12}\left(q_{i-2}-2 q_{i-1}+q_{i}\right)^{2}+\frac{1}{4}\left(q_{i-2}-4 q_{i-1}+3 q_{i}\right)^{2}, \\
& \beta_{2}=\frac{13}{12}\left(q_{i-1}-2 q_{i}+q_{i+1}\right)^{2}+\frac{1}{4}\left(q_{i-1}-4 q_{i+1}\right)^{2}, \\
& \beta_{3}=\frac{13}{12}\left(q_{i}-2 q_{i+1}+q_{i+2}\right)^{2}+\frac{1}{4}\left(3 q_{i}-4 q_{i+1}+q_{i+2}\right)^{2} .
\end{aligned}
$$

More details about the WENO scheme can be found elsewhere. $^{12-14}$

Equation (2) is discretized using first-order time marching, with a semi-implicit in time scheme for the diffusion terms, and the second-order Adams-Bashforth scheme for convection and central spatial differencing for diffusion. The WENO scheme is only used for the convection terms in the region around the flow/solid and flow/porous interfaces. Continuity is enforced by applying a divergence operator to both sides of Eq. (2) and invoking the incompressibility condition of Eq. (3) to obtain a Poisson equation for the 
pressure. The Poisson equation is solved with a fast solver. ${ }^{20}$ A more detailed explanation of the computational scheme and the related IB method can be found in Refs. 10 and 15 . It should be noted that, since we intend to capture all of the flow fluctuations, no turbulence models are used in the simulation, and the two-dimensional set up of the problem makes such direct simulation possible with the current computer power limit.

\section{RESULTS AND DISCUSSION}

We define the diameter of the upstream circular cylinder as $D$ in Fig. 1; the diameter of the bare microphone is $0.25 D$, a size selected to be proportional to some practical cases and compatible with the current simulation Reynolds number as explained further later. The ellipse windscreen shape is represented by the ellipse equation, $x^{2} / a^{2}+y^{2} / b^{2}=1$. We selected three typical ellipse shapes: the first case is a circular cylinder with $a=0.5 D, b=0.5 D$; the second case is a horizontally oriented elliptical cylinder with $a=1.0 D$, $b=0.25 D$; the third case is a vertically oriented elliptical cylinder with $a=0.25 D, b=1.0 D$. The rectangle windscreen shape is represented by the equation, $x^{2} / a^{2} \leq 1, y^{2} / b^{2} \leq 1$. We selected two typical rectangle shapes: the first case is a horizontal rectangle with $a=1.0 D, b=0.25 D$; the second case is a vertical rectangle with $a=0.25 D, b=1.0 D$. In the following discussion, we will call the circular windscreen case $\mathrm{C}$, the horizontal ellipse windscreen case $\mathrm{E} 1$, the vertical ellipse windscreen case E2, the horizontal rectangle windscreen case $\mathrm{R} 1$, and the vertical rectangle windscreen case R2. The distance, $L$, between the upstream cylinder center and the downstream windscreen center, is $8 D$. With these geometrical parameters, the region in which the WENO scheme is implemented is thus a rectangular region $12 D$ in length and $4 D$ in height. The distance ahead of the center of the upstream solid cylinder is $2 D$.

The Reynolds number for the cases presented here is 5000, which approximately corresponds to a windscreen with a diameter of $7.5 \mathrm{~cm}$, a bare microphone with a diameter of $1.875 \mathrm{~cm}$, and a speed of $1 \mathrm{~m} / \mathrm{s}$ for the incoming air. At this high-Reynolds number, a relatively broad spectrum of pressure fluctuations is generated with flow over the upstream circular cylinder, in contrast to a low-Reynolds number flow, where only very tonal pressure fluctuations, related to the von Karman vortex shedding frequency, are generated. The shape of the broadband spectrum of the unscreened case (to be shown shortly) is very similar to those of wind noise in the literature ${ }^{6-21}$ that follow the $-5 / 3$ frequency power decay of the spectrum of atmospheric turbulent pressure.

The three chosen values of dimensionless flow resistivity of the windscreen, 1,10 , and 100 , correspond to dimensional flow resistivity values of approximately 17,170 , and $1700 \mathrm{~Pa} \mathrm{~s} / \mathrm{m}^{2}$, respectively $\left[1 \sigma=\rho U / D=1.27\left(\mathrm{~kg} / \mathrm{m}^{3}\right) \cdot 1\right.$ $\left.(\mathrm{m} / \mathrm{s}) / 0.075(\mathrm{~m})=17\left(\mathrm{~Pa} \mathrm{~s} / \mathrm{m}^{2}\right)\right]$. In the simulation here, the grid size is 0.025 dimensionless units (in $D$ ) in both the $x$ and $y$ directions, and the time step is 0.0005 dimensionless units (in $D / U$ ) which satisfies the grid convergence and stability requirements for the computational scheme. ${ }^{15}$

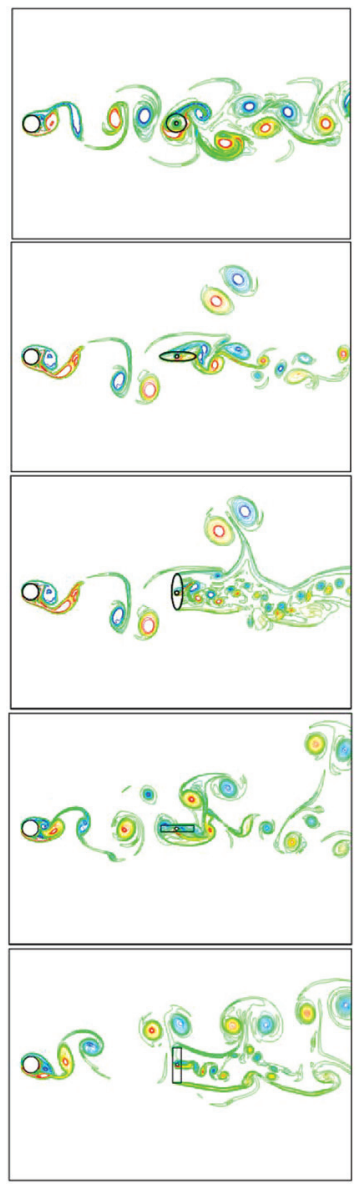

(a)

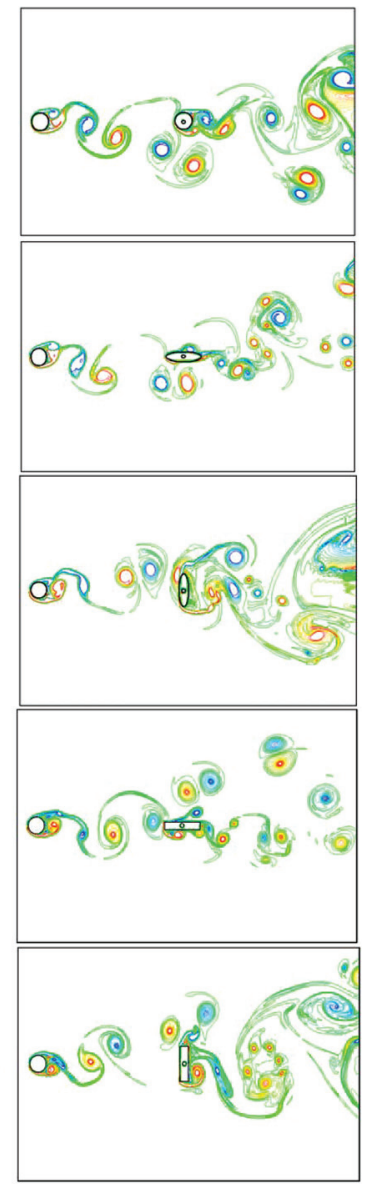

(b)
FIG. 2. (Color online) Vorticity contours of the flow field, for different shapes of windscreens (from top to bottom, C, E1, E2, R1, and R2) with flow resistivity of (a) $\sigma=17 \mathrm{~Pa} \mathrm{~s} / \mathrm{m}^{2}$ and (b) $\sigma=1700 \mathrm{~Pa} \mathrm{~s} / \mathrm{m}^{2}$.

We first investigate the whole flow field as shown by vorticity contours in Fig. 2 for the two extreme flow resistivities, $\sigma=17 \mathrm{~Pa} \mathrm{~s} / \mathrm{m}^{2}$ and $\sigma=1700 \mathrm{~Pa} \mathrm{~s} / \mathrm{m}^{2}$, with five different windscreen shapes (from top to bottom, C, E1, E2, R1, and R2). In Fig. 2, the vorticity contour range is from -10 (clockwise sense of rotation, represented with the dark blue color) to 10 (counter-clockwise sense of rotation, represented with the red color). The upstream cylinder, the wind screen, and the microphone are represented in the figure with black circles. While there is no flow inside the solid objects, some flow infiltrates the porous windscreen. The vortical structures shed from the upstream cylinder are diffused by the porous windscreen. When the flow resistivity is high $\left(\sigma=1700 \mathrm{~Pa} \mathrm{~s} / \mathrm{m}^{2}\right)$, little flow permeates the porous windscreen, and when the flow resistivity is low $\left(\sigma=17 \mathrm{~Pa} \mathrm{~s} / \mathrm{m}^{2}\right)$, the flow permeates the porous windscreen more easily.

In comparing different shape screens in Fig. 2, it can be seen that the fewest vortices are generated in the wake of the horizontal ellipse, while the most vortices are generated in the wake of the vertical ellipse and rectangle. The quantity of vortices in the wake of the circular and horizontal rectangular windscreens lies between these three cases. This is because the horizontal ellipse has the smallest area projected in the flow direction, while the vertical ellipse and rectangle have the largest area, so that stronger wakes are generated. 

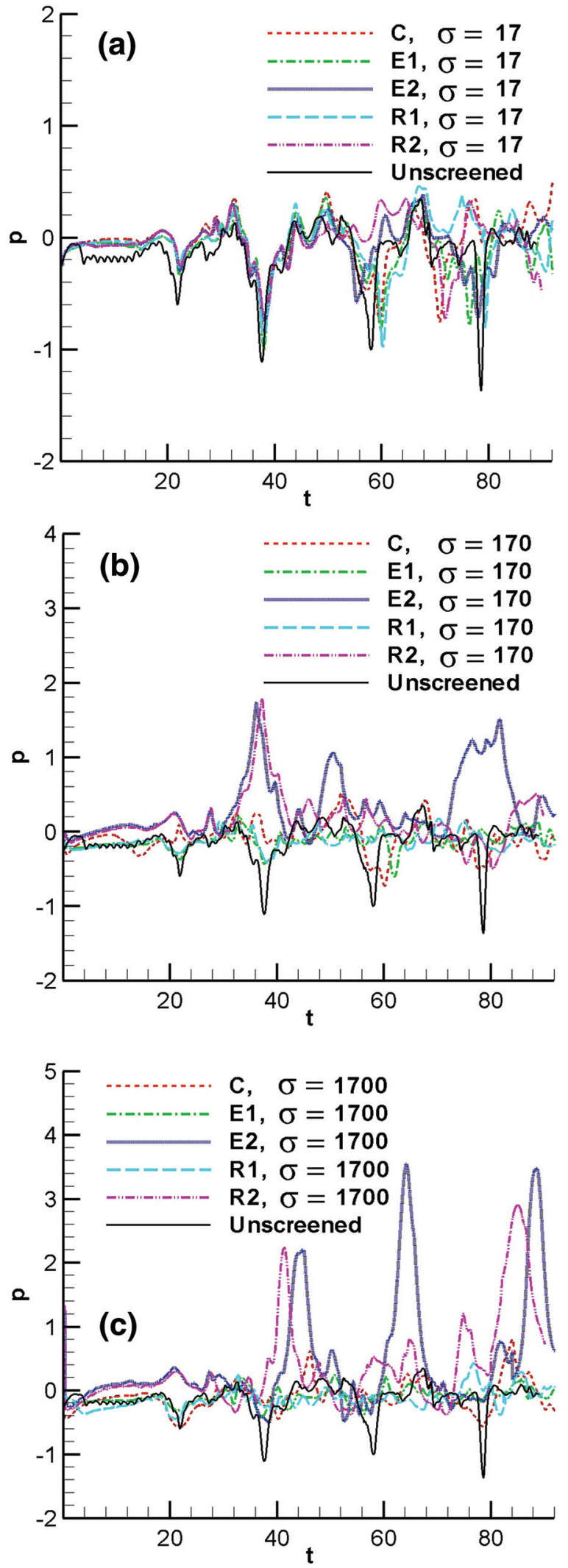

FIG. 3. (Color online) Pressure histories at the center of the windscreen for different shape windscreens (all values are dimensionless): (a) $\sigma=17 \mathrm{~Pa}$ $\mathrm{s} / \mathrm{m}^{2}$, (b) $\sigma=170 \mathrm{~Pa} \mathrm{~s} / \mathrm{m}^{2}$, and (c) $\sigma=1700 \mathrm{~Pa} \mathrm{~s} / \mathrm{m}^{2}$.

This directly affects the pressure fluctuations detected at the microphone as shown in Fig. 3, although the shape and porosity of the windscreen can influence sensed pressure fluctuations from both incoming turbulence as well as the wake after the windscreen, due to vortices diffused by the windscreen.

Figure 3 presents the pressure time histories of the center point of the microphone. We look at the histories of each one of the five shapes of windscreens under different flow resistivities $\left(\sigma=17,170\right.$, and $\left.1700 \mathrm{~Pa} \mathrm{~s} / \mathrm{m}^{2}\right)$, along with the unscreened case and compare the histories of the same flow resistivity under different windscreen shapes in Fig. 3. The dimensionless pressure and time in Fig. 3 are multipliers of $\rho U^{2}$ and $D / U$, respectively, as explained earlier.

The pressure time histories show that the fluctuation magnitude of the unscreened center pressure is higher than the $\mathrm{C}, \mathrm{E} 1$, and $\mathrm{R} 1$ cases among all of the three resistivity cases. Hence, for the circular, horizontal ellipse, and rectangle windscreens, there is at least some WNR. Although it is difficult to recognize the reduction from the time histories (which will be clear when we plot these results in the spectral domain in the following sections), it is still discernable that the medium flow resistivity material $\left(\sigma=170 \mathrm{~Pa} \mathrm{~s} / \mathrm{m}^{2}\right)$ provides more noise reduction than the low or high flow resistivity materials for these cases. For the E2 and R2 cases, only the low resistivity windscreen shows some noise reduction effect. The medium resistivity windscreen provides a little noise reduction, while the high resistivity windscreen increases the pressure fluctuations, which indicates a strong interaction between the upstream turbulence structure and the vertical ellipse windscreen in the E2 and R2 cases, as is evident in the flow fields shown in Fig. 2.

For the low resistivity cases in Fig. 3(a), the pressure fluctuation magnitudes are very similar on average among these five different shapes. Hence the low-resistivity windscreens provide very modest WNR. For the medium and high resistivity cases in Figs. 3(b) and 3(c), the C, E1, and R1 shapes reduce the pressure fluctuations significantly in comparison to the unscreened case; the E2 and R2 shapes show large magnitudes of pressure fluctuations, which means that wind noise is increased by the vertical ellipse and rectangle windscreens.

Next, we compare spectral levels for the various screens. The spectrum level (SL) is defined as $10 \log _{10}\left[\Delta f_{r} s_{p}(f) / p_{r}^{2}\right]$, where $\Delta f_{r}$ is the reference bandwidth of $1 \mathrm{~Hz}, P_{r}$ is the reference sound-pressure level of $20 \mu \mathrm{Pa}$, and $S_{p}(f)$ is the power spectral density (PSD) of the pressure at the microphone center. As shown in Fig. 3, the pressure time series data represent a turbulent and chaotic signal in the time domain. Therefore, when transforming the data to the spectral domain, windowing must be applied to reduce discontinuities at the ends of the time series. The most accurately resolved frequency range of a whole set of data is thus between $1 /\left(\Delta t N_{w}\right)$ and $1 /(2 r \Delta t)$, where the number of time series data points of each window is $N_{w}$, the number of sets of windowed data is $r$, and the time step is $\Delta t^{22}$ In this paper, the Blackman-Harris window with a size of $N_{w}=65536$ is used, and the overlapping percentage is $90 \%$ with $r=21$, resulting in an accurate frequency range from 0.4 to $630 \mathrm{~Hz}$.

Figures 4(a)-4(c) compare the SL of the five shapes of windscreens for each of the three flow resistivities $(\sigma=17$, 170 , and $1700 \mathrm{~Pa} \mathrm{~s} / \mathrm{m}^{2}$ ), along with the bare microphone case. All data shown in Fig. 4 are dimensionalized by multiplying the unit pressure of $\rho U^{2}=1.27 \mathrm{~Pa}$ and the unit 

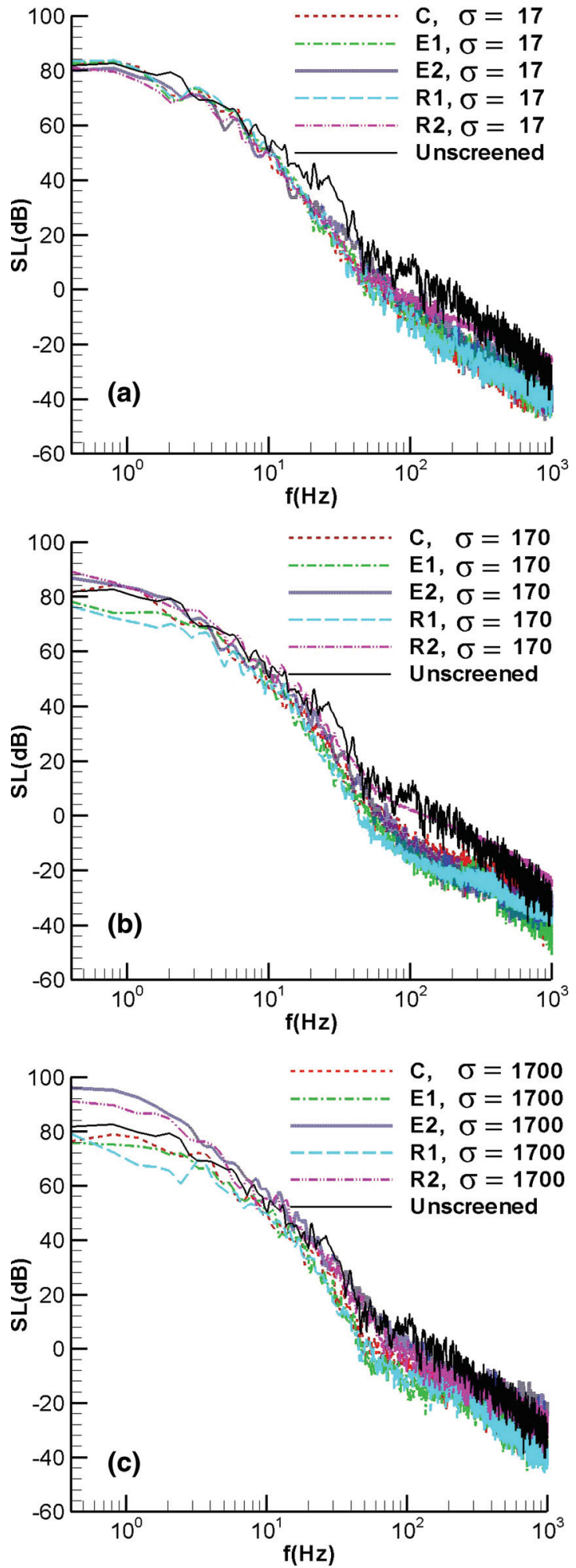

FIG. 4. (Color online) SL at the center of the windscreen for different shape windscreens (all values are dimensional): (a) $\sigma=17 \mathrm{~Pa} \mathrm{~s} / \mathrm{m}^{2}$, (b) $\sigma=170 \mathrm{~Pa}$ $\mathrm{s} / \mathrm{m}^{2}$, and (c) $\sigma=1700 \mathrm{~Pa} \mathrm{~s} / \mathrm{m}^{2}$.

frequency of $U / D=13.33 \mathrm{~Hz}$. It should be noted that all SL curves have a broad spectral character, as does realistic wind turbulence. While the results presented here are for a 1Ddiameter-sized upstream circular cylinder, we also tested other sizes of circular cylinders. Unless the circular cylinder size is so small that the effective Reynolds number is in a non-turbulent regime, turbulence generated by cylinders larger than 1D always exhibits a broadband spectrum and very similar to those shown here.

For the circular cylinder, horizontal ellipse and rectangle windscreens (C, E1, and R1), there is little noise reduction effect below the frequency of $10 \mathrm{~Hz}$ for the low flow resistivity ( $\sigma=17 \mathrm{~Pa} \mathrm{~s} / \mathrm{m}^{2}$ ) cases. However, there is significant noise reduction between frequencies of 20 and $400 \mathrm{~Hz}$. Furthermore, for the $\mathrm{C}$ and $\mathrm{R} 1$ cases within the $10-60 \mathrm{~Hz}$ range, Fig. 4 also shows that the three different resistivities for the porous windscreen material do not significantly impact the noise reduction response. When the flow resistivity increases to medium or high $\left(\sigma=170\right.$ or $\left.1700 \mathrm{~Pa} \mathrm{~s} / \mathrm{m}^{2}\right)$, Figs. 4(b) and 4(c) show that the noise reduction effect improves in the frequency range between 30 and $100 \mathrm{~Hz}$ in this study. This trend is more significant for the E1 and R1 cases. For the $\mathrm{C}$ cases, the low flow resistivity produces more noise reduction than medium and high flow resistivity over most of the frequency range.

In contrast, the E2 and R2 cases in Fig. 4(c), with the high flow resistivity windscreen, show very poor noise reduction behavior. In fact, noise levels are increased compared to those of the bare microphone. For medium flow resistivity $\left[\sigma=170 \mathrm{~Pa} \mathrm{~s} / \mathrm{m}^{2}\right.$, Fig. 4(b)], the vertical ellipse and rectangle windscreens provide a small noise reduction in the frequency range from 50 to $200 \mathrm{~Hz}$.

The above results on high resistivity windscreens do not contradict to the non-porous windscreen results in Ref. 21. In that study, the spectrum of pressure fluctuation did peak at the vortex shedding frequency, which agrees with the fact that high flow resistivity causes wake turbulence. However, as the wake frequency range was outside of the interested frequency range in that study, the wake did not affect the performance of the windscreen in their cases.

Finally, in order to clearly quantify the WNR effect, we investigate WNR levels between the unscreened microphone and the screened microphone, defined as $\mathrm{WNR}=\mathrm{SL}_{1}-\mathrm{SL}_{2}$, where $\mathrm{SL}_{1}$ is the spectrum level in the unscreened microphone center, and $\mathrm{SL}_{2}$ is the spectrum level in the screened microphone center. Table I lists the most significant results. In Tables I(a)-I(c), the results are grouped by like resistivity values, but with different windscreen shapes. It should be noted that the noise levels in Table I may not necessarily match real cases in the absolute sense; however, Table I summarizes the important trends in the simulation results that reveal the effect of WNR in a relative sense. The maximum WNR is around $30 \mathrm{~dB}$, which is a very significant WNR in the cases of C, E1, and R1, and occurs with medium flow resistivity at around $188 \mathrm{~Hz}$. The largest noise increase, with a WNR of approximately $-13 \mathrm{~dB}$, occurs for the vertical ellipse (E2) and rectangular (R2) windscreens with a high flow resistivity porous material in the frequency range of a few hertz to $100 \mathrm{~Hz}$. Therefore the windscreen shape affects the WNR behavior significantly, although flow resistivity and frequency range also have an influence.

The overall behaviors of the WNR between the circular cylinder, horizontal ellipse, and horizontal rectangle windscreens are similar, for which the lowest flow resistivity windscreens $\left(\sigma=17 \mathrm{~Pa} \mathrm{~s} / \mathrm{m}^{2}\right)$ have less noise reduction than the other two higher flow resistivity screens $(\sigma=170$ and 
TABLE I. WNR (in $\mathrm{dB}$ ) between unscreened and screened microphones with different shapes of windscreens.

\begin{tabular}{|c|c|c|c|c|c|}
\hline Frequency/(Hz)/shape & $\mathrm{C}$ & E1 & E2 & R1 & $\mathrm{R} 2$ \\
\hline \multicolumn{6}{|c|}{ (a) Resistivity $\sigma=17 \mathrm{~Pa} \mathrm{~s} / \mathrm{m}^{2}$} \\
\hline 15.4 & 13.7 & 11.1 & 16.3 & 12.9 & 12.2 \\
\hline 25.6 & 19.1 & 18.8 & 17.9 & 20.4 & 16.0 \\
\hline 53.3 & 14.5 & 20.4 & 13.2 & 21.4 & 13.4 \\
\hline 88.3 & 18.6 & 15.4 & 16.4 & 18.7 & 14.5 \\
\hline 188.1 & 27.4 & 24.2 & 17.7 & 23.1 & 15.2 \\
\hline 261.6 & 21.4 & 14.8 & 20.9 & 20.5 & 10.6 \\
\hline \multicolumn{6}{|c|}{ (b) Resistivity $\sigma=170 \mathrm{~Pa} \mathrm{~s} / \mathrm{m}^{2}$} \\
\hline 15.4 & 8.2 & 16.7 & 5.2 & 16.9 & -3.7 \\
\hline 25.6 & 16.0 & 22.6 & 9.4 & 22.3 & 7.2 \\
\hline 53.3 & 19.1 & 18.0 & -4.1 & 24.7 & -6.6 \\
\hline 88.3 & 20.1 & 20.9 & 14.0 & 26.4 & 5.8 \\
\hline 188.1 & 29.3 & 28.7 & 18.7 & 27.6 & -4.5 \\
\hline 261.6 & 18.6 & 22.3 & 14.8 & 21.6 & -8.1 \\
\hline \multicolumn{6}{|c|}{ (c) Resistivity $\sigma=1700 \mathrm{~Pa} \mathrm{~s} / \mathrm{m}^{2}$} \\
\hline 15.4 & 8.6 & 12.8 & -4.4 & 10.1 & -10.3 \\
\hline 25.6 & 15.5 & 13.2 & 7.0 & 17.2 & 6.8 \\
\hline 53.3 & 13.8 & 22.6 & -10.2 & 19.6 & -7.5 \\
\hline 88.3 & 14.8 & 21.6 & 3.9 & 24.9 & -4.8 \\
\hline 188.1 & 21.9 & 19.0 & -2.2 & 20.9 & -5.4 \\
\hline 261.6 & 12.3 & 19.9 & -4.3 & 15.5 & 7.7 \\
\hline
\end{tabular}

$1700 \mathrm{~Pa} \mathrm{~s} / \mathrm{m}^{2}$ ); the circular windscreens perform somewhat better than the horizontal ellipse and rectangle windscreens. The vertical ellipse and rectangle windscreens are ineffective, especially for the high flow resistivity cases, when additional self-noise is generated.

For the circular cylinder cases in Table I and Fig. 4, the medium flow resistivity materials are more effective in the high-frequency range $(f>50 \mathrm{~Hz})$, while the low-resistivity material has more effect in the low-frequency range $(f<50$ $\mathrm{Hz}$ ). For the horizontal ellipse and rectangle cases, the medium resistivity is the most effective among the three resistivities over almost all of the frequency range. For the vertical ellipse and rectangle cases, the low-resistivity material has more effect in most of the frequency ranges than the other two, while the high resistivity windscreen adversely affects noise reduction.

In summary, the medium flow resistivity windscreens $\left(\sigma=170 \mathrm{~Pa} \mathrm{~s} / \mathrm{m}^{2}\right)$ perform best and achieve the highest WNR. The circular cylinder, horizontal ellipse, and rectangle windscreens behave similarly overall, while the vertical ellipse and rectangle windscreens do not provide significant WNR and, in fact, if made with high flow resistivity materials, they increase the wind noise in most of the frequency range.

\section{CONCLUSION}

This paper examined WNR by porous windscreens of three different shapes. A WENO scheme, implemented with an IB method, was employed to improve the accuracy at the interface between the open air and porous medium. A coupled computation including flow both outside and inside the windscreen was used. Different shape windscreens, with varying flow resistivities, were tested under a high-Reynolds-number wind turbulence condition, which was generated by an upstream solid cylinder. The simulation results show that the horizontal ellipse windscreen with medium flow resistivity is most effective in WNR, although the circular cylinder and horizontal rectangle windscreens produce similar noise reduction in most of the frequency range. The vertical ellipse and rectangle windscreens provide some wind reduction with low flow resistivity, but increase the wind noise when the flow resistivity of the windscreen material is higher. The mechanism of WNR is the ability of windscreens to diffuse the vortical structures in the turbulent flow. The increased wind noise for the vertical ellipse and rectangle windscreens is attributable to the relatively large, projected area perpendicular to the flow direction, which generates strong wake vortices and thus self-noise.

While it should be kept in mind that the above conclusions are based on two-dimensional numerical simulation results at Reynolds numbers considerably smaller than typical atmospheric turbulence, they do capture the qualitative physics of a porous windscreen interacting with a high-Reynolds number flow. More realistic simulations, which might provide useful quantitative guidance in the WNRs attainable with three-dimensional windscreens, are yet to be conducted.

\section{ACKNOWLEDGMENTS}

This research was partly supported by the U.S. Army Engineering Research and Development Center under contract W913E7-07-C-0004 when the first two authors were at Kansas State University.

${ }^{1}$ M. Strasberg, "Dimensional analysis of windscreen noise," J. Acoust. Soc. Am. 83(2), 544-548 (1988).

${ }^{2}$ S. Morgan and R. Raspet, "Investigation of the mechanisms of low-frequency wind noise generation outdoors," J. Acoust. Soc. Am. 92(2), 1180-1183 (1992).

${ }^{3}$ Z. C. Zheng, "A computational study of the flow/acoustic mechanisms in screened microphones," in Proceedings of the ASME 2001 Fluids Engineering Division Summer Meeting, Paper number FEDSM2001-18204, New Orleans, LA (May 29 to June 1, 2001).

${ }^{4}$ Z. C. Zheng and B. K. Tan, "Reynolds number effects on flow/acoustic mechanisms in spherical windscreens," J. Acoust. Soc. Am. 113(1), 161166 (2003).

${ }^{5}$ M. S. Morgan, "An investigation of the sources and attenuation of wind noise in measurement microphones," $\mathrm{Ph}$. D. dissertation, Department of physics and Astronomy, University of Mississippi, PARGUM Report, 1993, pp. 93-101.

${ }^{6}$ R. Raspet, J. Webster, and K. Dillion, "Framework for wind noise studies," J. Acoust. Soc. Am. 119(2), 834-843 (2006).

${ }^{7}$ G. P. van den Berg, "Wind-induced noise in a screened microphone," J. Acoust. Soc. Am. 119(2), 824-833 (2006).

${ }^{8}$ V. E. Ostashev, D. K. Wilson, L. Liu, D. F. Aldridge, N. P. Symons, and D. H. Marlin, "Equations for finite-difference, time-domain simulation of sound propagation in moving inhomogeneous media and numerical implementation," J. Acoust. Soc. Am. 117(2), 503-517 (2005).

${ }^{9}$ D. K. Wilson, S. L. Collier, V. E. Ostashev, D. F. Aldridge, N. P. Symons, and D. H. Marlin, "Time-domain modeling of the acoustic impedance of porous surfaces," Acta Acust. Acust. 92, 965-975 (2006).

${ }^{10} \mathrm{Y}$. Xu, Z. C. Zheng, and D. K. Wilson, "Simulation of turbulent wind noise reduction by porous windscreens using high-order schemes," J. Comput. Acoust. 18(4), 321-334 (2010).

${ }^{11}$ C. Zwikker and C. W. Kosten, Sound Absorbing Materials (Elsevier, New York, 1949), pp. 18-24.

${ }^{12}$ A. Harten, B. Engquist, S. Osher, and S. Chakravarthy, "Uniformly high order accurate non-oscillatory schemes, III," J. Comput. Phys. 71(2), 231303 (1987).

${ }^{13}$ C. W. Shu and S. Osher, "Efficient implementation of nonoscillatory shock capturing schemes,” J. Comput. Phys. 83, 32-78 (1988). 
${ }^{14}$ Y. Cho, J. Chopra, and P. J. Morris, "Immersed boundary method for compressible high-Reynolds number viscous flow around moving bodies," in 45th AIAA Aerospace Sciences Meeting, Paper number AIAA2007-125, Reno, NV (January 8-11, 2007).

${ }^{15} \mathrm{~N}$. Zhang and Z. C. Zheng, "An improved direct-forcing immersed-boundary method for finite difference applications," J. Comput. Phys. 221(1), 250-268 (2007).

${ }^{16}$ G. H. Goedecke, V. E. Ostashev, D. K. Wilson, and H. J. Auvermann, "Quasi-wavelet model of von Karman spectrum of turbulent velocity fluctuations," Boundary-Layer Meteorol. 112, 33-56 (2004).

${ }^{17}$ D. K. Wilson, V. E. Ostashev, G. H. Goedecke, and H. J. Auvermann, "Quasi-wavelet calculations of sound scattering behind barriers," Appl. Acoust. 65, 605-627 (2004).
${ }^{18}$ R. H. Kraichnan, "Pressure fluctuations in turbulent flow over a flat plate," J. Acoust. Soc. Am. 28(3), 378-390 (1956).

${ }^{19}$ D. K. Wilson, V. E. Ostashev, S. L. Collier, N. P. Symons, D. F. Aldridge, and D. H. Marlin, "Time-domain calculations of sound interactions with outdoor ground surfaces," Appl. Acoust. 68(2), 173-200 (2007).

${ }^{20}$ P. N. Swarztrauber and R. A. Sweet, "Algorithm 541: Efficient Fortran subprograms for the solution of separable elliptic partial differential equations [D3]," ACM Trans. Math. Softw. 5(3), 352-364 (1979).

${ }^{21}$ Q. A. Shams, A. J. Zukerwar, and B. S. Sealey, "Compact nonporous windscreen for infrasonic measurements," J. Acoust. Soc. Am. 118(3), $1335-1340$ (2005)

${ }^{22}$ J. C. Kaimal and J. J. Finnigan, Atmospheric Boundary Layer Flows (Oxford University, New York, 1994), pp. 269-272. 